\title{
A modified method for the culture of naturally HPV-infected high-grade cervical intraepithelial neoplasia keratinocytes from human neoplastic cervical biopsies
}

\author{
YU-ZHEN LIU ${ }^{1}$, TIAN-TIAN WANG ${ }^{1}$ and YOU-ZHONG ZHANG ${ }^{2}$ \\ ${ }^{1}$ Department of Obstetrics and Gynecology, Affiliated Hospital of Weifang Medical University, Weifang, Shandong 261041; \\ ${ }^{2}$ Department of Obstetrics and Gynecology, Qilu Hospital, Shandong University, Jinan, Shandong 250012, P.R. China
}

Received February 1, 2015; Accepted December 14, 2015

DOI: $10.3892 / 01.2016 .4096$

\begin{abstract}
Few studies on cervical intraepithelial neoplasia (CIN) keratinocyte cultures are available due to the numerous technical and methodological problems associated with the in vitro cultivation of these cells. The present study investigated an applicable and effective method for the in vitro cultivation of high-grade CIN keratinocytes from human neoplastic cervical biopsies. Human neoplastic cervical tissue sections were obtained and digested using type I collagen in order to dissociate the cells. The cells were seeded in tissue culture plastic plates that were coated with rat tail collagen type I and contained modified keratinocyte serum-free medium (K-SFM) supplemented with $5 \%$ fetal bovine serum. The medium was replaced with K-SFM on days 3, 5 and 7, respectively. The unattached cells were recovered and the cell viability was determined accurately using the Trypan Blue exclusion method. The expression of keratin 14 (K14), keratin 19 (K19), keratin 17 (K17) and P63 was assayed using immunofluorescence in order to identify the presence of CIN keratinocytes. The present results indicated that the attachment rate of CIN keratinocytes significantly increased between $56.75 \pm 1.76 \%$ on day 3 and $77.09 \pm 3.55 \%$ on day 5 , and became relatively stable between days 5 and 7 . The cell viability significantly decreased between $83.00 \pm 0.50 \%$ on day 5 and $68.17 \pm 1.04 \%$ on day 7 . The passaged CIN keratinocytes maintained the original unequally sized, abnormally shaped morphology and did not undergo differentiation. In
\end{abstract}

Correspondence to: Dr You-Zhong Zhang, Department of Obstetrics and Gynecology, Qilu Hospital, Shandong University, 107 Wenhua West Road, Jinan, Shandong 250012, P.R. China E-mail: zhangyouzhong@sdu.edu.cn

Abbreviations: NUC, normal uterine cervix; CIN, cervical intraepithelial neoplasia; K-SFM, keratinocyte serum-free medium; $\mathrm{HPV}$, human papillomavirus

Key words: cervical intraepithelial neoplasia, keratinocyte, epithelial cells, primary cell culture, cell attachment, cell viability addition, the passaged CIN keratinocytes exhibited the same human papilloma virus (HPV) genotype that was detected in the original primary cells. K14 and K19 were expressed in the majority of the normal and CIN keratinocytes, whereas K17 and P63 were expressed only in high-grade CIN keratinocytes. The present study proposes a simple and practical method for rapidly obtaining highly purified naturally HPV-infected high-grade CIN keratinocytes from small neoplastic cervical tissues, and provides an appropriate first medium change time for the primary culture of CIN keratinocytes.

\section{Introduction}

Cervical cancer is the most common gynecological cancer worldwide and is usually preceded by a long phase of pre-malignant disease $(1,2)$. These pre-malignant changes represent a spectrum of histological abnormalities ranging between cervical intraepithelial neoplasia (CIN) I (mild dysplasia), CIN II (moderate dysplasia) and CIN III (severe dysplasia/carcinoma in situ) $(3,4)$. In the majority of patients, CIN I will regress spontaneously and is not usually precancerous; however, $20-45 \%$ of untreated CIN II and III lesions will persist and progress to cervical cancer $(5,6)$. Therefore, CIN II and CIN III are considered to be high-grade precancerous lesions. Previous studies have established that the majority of high-grade CIN cases are caused by persistent oncogenic human papilloma virus (HPV) infection, and a long latency period of viral infection is required for CIN progression (7-9). However, viruses do not metabolize, and require a host cell to make new products. Therefore, HPV may not be cultured in a laboratory outside of a host cell (10). The culture of naturally HPV-infected CIN keratinocytes has significant value for the exploration of the biological behavior of high-grade CIN in vitro and in vivo.

Few studies on CIN keratinocyte cultures and on the biological characteristics of CIN keratinocytes are available due to associated technical and methodological issues (11). At present, only two studies in the literature describe techniques for the isolation and cultivation of CIN keratinocytes from neoplastic cervical biopsies. In the study by Stanley (12), entire cervical tissues were micro-dissected and the keratinocytes that migrated from the explants were rescued and 
subsequently seeded onto a feeder layer of inactivated murine fibroblasts $(11,12)$. The major issue with this study was the use of the irradiated mouse 3T3 feeder layer cells, which were regarded as a potential risk due to the possible transfer of disease, such as animal viruses or prions. In addition, the low number of CIN keratinocytes rescued was frequently associated with contaminant human fibroblasts, which rapidly overgrew the epithelial cells in culture (11-13). Additionally, the growth of the keratinocytes was constrained due to the limited space during the course of culture. Bononi et al (11) reported a method that digested CIN tissue fragments overnight using collagenase II, and the derived fibroblasts and keratinocytes were co-cultured in Dulbecco's modified Eagle's medium (DMEM)-F12 containing 10\% fetal bovine serum (FBS) (11). The limitations of the aforementioned study were that the protocol used was relatively complex and the culturing period was long. Additionally, the increased concentration of FBS used in these two studies may induce cell differentiation and contamination of human fibroblasts (11,14-16).

In the present study, the explant tissue culture and digestion culture methods were combined in order to establish a brief and practical method for culturing naturally HPV-infected high-grade CIN keratinocytes, and plenty of isolated cells were obtained. Additionally, the culture medium and culture plastic plates were modified, as reported in a preliminary study (17). The first medium change time was prolonged and the cell viability and cell attachment rate were monitored on days 3, 5 and 7. The cultured keratinocytes were identified using immunofluorescence, which was used to determine the expression of epithelial and cervical markers.

\section{Materials and methods}

CIN specimens. Small tissue fragments $\left(2-4 \mathrm{~mm}^{3}\right)$ were collected from 3 patients with high-grade neoplastic cervical lesions that had previously undergone conization during a colposcopic examination, through the application of $5 \%$ acetic acid and Lugol's iodine solution. The CIN specimens were selected and divided by the gynecologist during surgery, and classified by pathologists according to international criteria $(11,18)$. The study was approved by the Ethics Committee of Weifang Medical University (Weifang, China), and informed written consent was obtained from all patients. Three normal uterine cervix (NUC) tissue fragments were provided as controls. Following removal, the specimens were maintained at $4^{\circ} \mathrm{C}$ in a transporting medium that contained HyClone DMEM-F12 (GE Healthcare, Logan, UT, USA), $10 \%$ HyClone FBS (GE Healthcare) and antibiotics $(10 \mu \mathrm{g} / \mathrm{ml}$ amphotericin B; 200 international units (IU)/ml penicillin; 200 mg/ml streptomycin; Beijing Solarbio Science \& Technology Co., Ltd., Beijing, China), and transferred to the laboratory within $2 \mathrm{~h}$.

Isolation and primary culture of high-grade CIN keratinocytes. The tissue fragments were washed twice for $5 \mathrm{~min}$ in phosphate-buffered saline (PBS) that contained penicillin-streptomycin and amphotericin $\mathrm{B}$, in order to remove possible infections. The isolation of CIN was conducted as described in a previous study (17). Small pieces of tissue were digested using $0.2 \%$ Gibco type I collagenase solution
(Thermo Fisher Scientific, Inc., Waltham, MA, USA) in a gas bath thermostatic oscillator (THZ-82B, Jintan Medical Instrument Factory, Jintan, China) at $37^{\circ} \mathrm{C}$ and $200 \mathrm{r} / \mathrm{min}(0.9 \mathrm{x} \mathrm{g}$ for $40 \mathrm{~min}$. Unlike a previous study (17), the isolated cells in the present study were not filtered through a mesh. The cells were seeded directly into 6-well flat-bottomed culture plastic plates that were coated with $2 \mu \mathrm{g} / \mathrm{cm}^{2}$ rat tail collagen type I (Sigma-Aldrich, St. Louis, MO, USA), and incubated at room temperature overnight in a medium that contained Gibco keratinocyte serum-free medium (K-SFM; Thermo Fisher Scientific, Inc.), 5\% FBS and antibiotics (2.5 $\mu \mathrm{g} / \mathrm{ml}$ amphotericin $\mathrm{B} ; 100 \mathrm{IU} / \mathrm{ml}$ penicillin; $100 \mathrm{mg} / \mathrm{ml}$ streptomycin) at a cell density of $7-10 \times 10^{4}$ cells $/ \mathrm{cm}^{2}$. The cells were cultured in a $\mathrm{CO}_{2}$ incubator at $37^{\circ} \mathrm{C}$ and were allowed to attach for 3 days. An inverted microscope and photo acquisition system (DMI6000; Leica Microsystems, Wetzlar, Germany) was used to monitor the cell growth and morphology.

Cell attachment calculation and cell viability determination. The Trypan Blue (Jimi Biotech Company Ltd., Shanghai, China) exclusion method was used to accurately determine the cell viability subsequent to isolation according to the manufacturer's protocols. On days 3,5 and 7, the serum-supplemented K-SFM was replaced with serum-free K-SFM in 2 of the wells. The unattached cells were recovered, the cell density was calculated using a dual-chamber hemocytometer (1103; Yancheng Hengtai Glass Instrument Factory, Yancheng, China), and the cell viability was determined using Trypan Blue exclusion. The medium was replaced twice a week during the following 10-14 days.

For the Trypan Blue staining, a $200 \mu 1$ cell suspension was aseptically transferred to a $1.5 \mathrm{ml}$ clear Eppendorf tube (Corning Incorporated, Corning, NY, USA) and incubated for $3 \mathrm{~min}$ at room temperature with an equal volume of $0.4 \%(\mathrm{w} / \mathrm{v})$ Trypan Blue solution that was prepared in buffered isotonic salt solution (Beijing Solarbio Science \& Technology Co., Ltd.). The cells were counted using a dual-chamber hemocytometer and a light microscope (CX21; Olympus Corporation, Toyko, Japan) (19). Viable and nonviable cells were recorded separately, and cell viability was calculated using the following formula: Viable cells $(\%)=[1$ - (number of blue cells / number of total cells)] x 100.

To calculate the rate of cell attachment, the following formula was used: Cell attachment $(\%)=[1$ - (cell density of unattached cells / cell density of seeding)] x 100 .

HPV detection and genotyping. A small proportion of the isolated CIN cells were used for DNA extraction simultaneously. HPV DNA was amplified using the L1 consensus HPV primers kit, (HybriBio Ltd., Hong Kong, China) as previously described (20). The internal controls included biotinylated primers, which amplified a 268-bp fragment of a human A-globin gene, as a positive control and biotin as a negative control. HPV genotyping was performed using an HPV GenoArray test kit (HybriBio, Hong Kong, China) as previously described $(20,21)$. The HPV blot contained 15 high-risk genotypes, including HPV16, 18, 31, 33, 35, 39, 45, 51, 52, $56,58,53,59,66$ and 68. HPV L1 and the internal control, human A-globin, confirmed that no HPV DNA contamination had taken place $(20,21)$. For the HPV genotyping of 


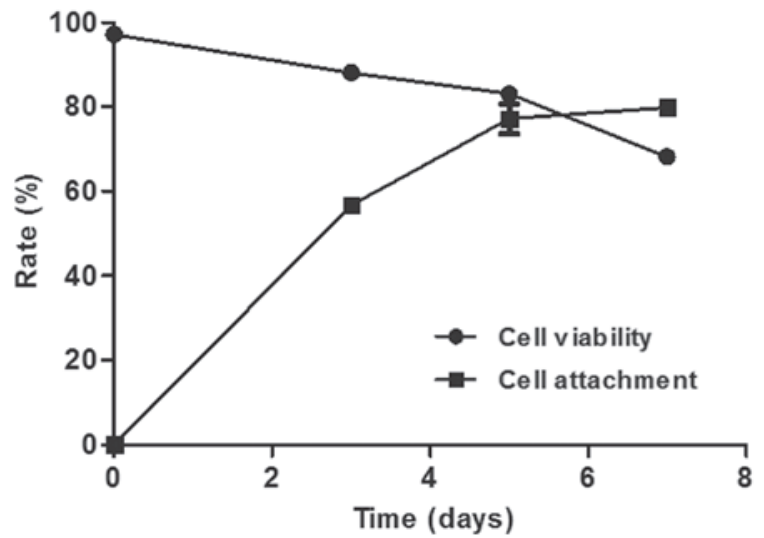

Figure 1. Cell viability and cell attachment rate of cervical intraepithelial neoplasia keratinocytes.

the sub-cultured CIN keratinocytes, a small number of the third-generation cells were subjected to DNA extraction and tested to determine the genotype.

Subculture and purification of high-grade CIN keratinocytes. The cells were sub-cultured when they reached $80 \%$ confluency by treatment with $0.25 \%$ Gibco Trypsin-ethylenediaminetetraacetic acid (Thermo Fisher Scientific, Inc.) for $5 \mathrm{~min}$. The cells were split at a ratio of 1:2 into dishes, all without the collagen type I coating, and the cells were incubated in the K-SFM to purify the CIN keratinocytes. This protocol was based on preliminary experiments, which showed that the attachment of sub-cultured cervical keratinocytes was not affected by collagen type I coating and grew well in K-SFM; however, the growth of fibroblasts was constrained in a medium with K-SFM alone (17).

Identification of high-grade CIN keratinocytes. The sub-cultured high-grade CIN keratinocytes were fixed using $100 \%$ ice-cold methanol for $15 \mathrm{~min}$ at $-20^{\circ} \mathrm{C}$ and permeabilized using $0.3 \%$ Triton in PBS for $30 \mathrm{~min}$. Subsequent to blocking the non-specific binding site with goat serum, the cells were incubated using various mouse or rabbit anti-human monoclonal antibodies (Beijing Solarbio Science \& Technology Co., Ltd.). To determine the epithelial and cervical markers, immunofluorescence staining was performed using mouse anti-human monoclonal keratin 14 (K14; \#LL001; sc-53253) and keratin 19 polyclonal (K19; \#N-13; sc-33119) antibodies (1:100 in PBS; Santa Cruz Biotechnology, Inc., Dallas, TX, USA). The rabbit anti-human monoclonal antibodies keratin 17 (K17; \#D73C7; $\mathrm{XP}^{\circledR}$ 4534P) and P63 (\#D2K8X; XP $\left.{ }^{\oplus} 13109 \mathrm{~T}\right)(1: 400$ in double-distilled water; Cell Signaling Technology, Inc., Danvers, MA, USA) were used to identify the high-grade CIN keratinocytes, which were considered to originate from cervical stem cells. Subsequent to washing three times with PBS, anti-mouse/rabbit immunoglobulin-fluorescein isothiocyanate (Jackson ImmunoResearch Laboratories, Inc., West Grove, PA, USA) was used as the secondary conjugated antibody. The nuclei of all of the cells, with the exception of those stained with the P63 antibody, were stained using 40,6-diamidino-2-phenylindole. High-grade CIN keratinocytes were detected using fluorescence microscopy (DM IRB; Leica Microsystems $\mathrm{GmbH}$, Wetzlar, Germany). The substitution of primary antibodies with PBS provided a negative control. HeLa cells (obtained from the American Type Culture Collection, Manassas, VA, USA) stained with K17 and P63 were cultured in HyClone RPMI-1640 medium (GE Healthcare) that contained $10 \% \mathrm{FBS}$, and provided a positive control. The percentage of cells that expressed the various keratin markers was quantified by counting 1,000 cells in four randomly selected fields. The aforementioned identification procedures were repeated three times.

Statistical analysis. Statistical analysis was performed using a one-way analysis of variance followed by the Student-Newman-Keuls comparison test. The data were analyzed using SPSS software (SPSS, Inc., for Windows, standard version 17.0; Chicago, IL, USA). $\mathrm{P}<0.05$ was considered to indicate a statistically significant difference.

\section{Results}

HPV DNA analysis of high-grade CIN. All of the neoplastic cervical specimens expressed high-risk HPV genotypes. Specifically, the CIN III specimens obtained in the present study expressed HPV-16, 31 and 45 . The third-generation cells were collected for HPV DNA extraction, and the same HPV genotypes were detected.

Cell viability of isolated CIN cells and unattached cells. The cell viability directly following the isolation was $97.17 \pm 1.04 \%$. On days 3,5 and 7, the unattached cells were recovered, and the cell viability was $88.00 \pm 0.50,83.00 \pm 0.50$ and $68.17 \pm 1.04 \%$, respectively. The cell viability significantly decreased between $83.00 \pm 0.50 \%$ on day 5 and $68.17 \pm 1.04 \%$ on day $7(\mathrm{P}=0.01$; Fig. 1).

Cell attachment rate of CIN cells. The cell density of high-grade CIN keratinocytes subsequent to isolation was $7-10 \times 10^{4}$ cells $/ \mathrm{cm}^{2}$. The attachment rate of high-grade CIN keratinocytes significantly increased between $56.75 \pm 1.76 \%$ on day 3 and $77.09 \pm 3.55 \%$ on day $5(\mathrm{P}=0.01)$, and became relatively stable between days 5 and $7(79.80 \pm 1.32 \% ; \mathrm{P}=0.655$; Fig. 1).

Growth behavior of CIN cells in primary culture. Daily cell monitoring through phase contrast microscopy indicated that only a few fibroblasts that exhibited a spindle-like shape were observed in disordered clusters or parallel bundle groups on day 3 (Fig. 2A), whereas high-grade CIN keratinocytes formed distinguishable colonies due to their unequally sized, abnormally shaped, enlarged nuclear and hyperchromasia morphology (Fig. 2A). When the two cell types began to proliferate, they maintained the growth and morphological characteristics. On days 12-14 of culturing, the fibroblasts had almost disappeared and the CIN keratinocytes reached $80 \%$ confluency (Fig. 2B). Additionally, a large variation was observed between high-grade CIN and NUC keratinocytes, which exhibited a uniform rounded or polygonal morphology (Fig. 2C). The experiments were repeated three times, and similar results were obtained. 

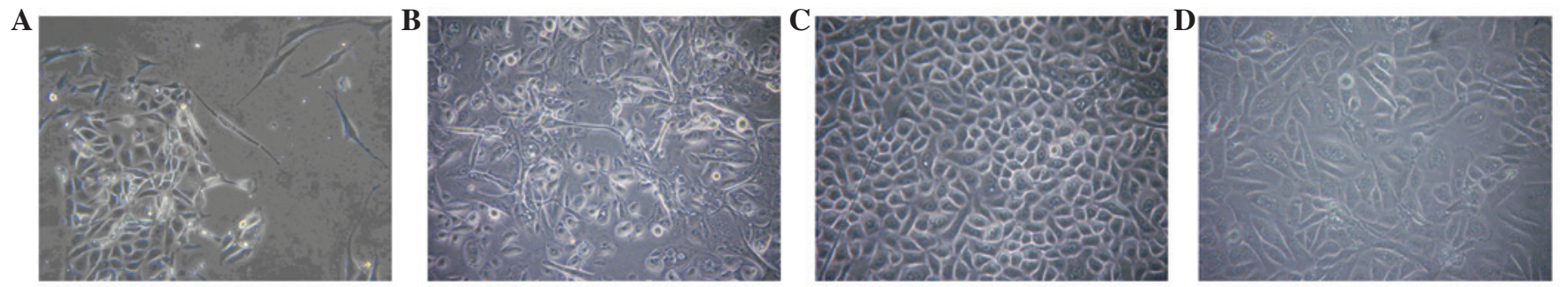

Figure 2. Cell morphology of keratinocytes. (A) High-grade CIN keratinocytes formed colonies and few fibroblasts exhibited a spindle-like shape on day 6 . (B) Primary cultured high-grade CIN keratinocytes exhibited an unequally sized, abnormally shaped, enlarged nuclear and hyperchromasia morphology on day 12. (C) Primary cultured NUC keratinocytes had a uniform rounded or polygonal morphology. (D) The first passaged CIN cells on day 10 maintained an unequally sized, abnormally shaped morphology. CIN, cervical intraepithelial neoplasia; NUC, normal uterine cervix.
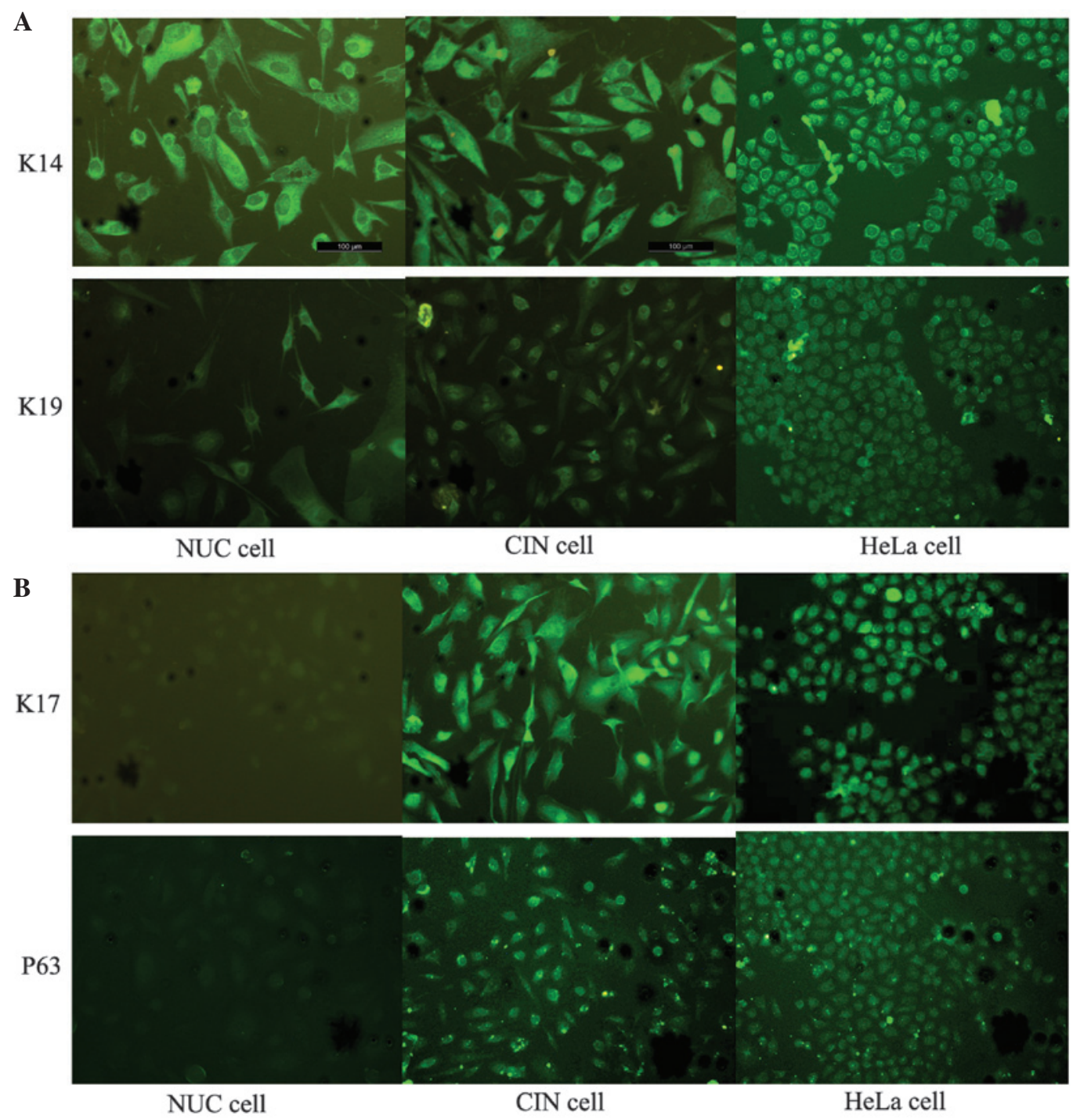

Figure 3. Immunofluorescence staining of passaged cervical epithelial cells and HeLa cells. (A) Cytoplasmic K14 and K19 expression in cultures of NUC, CIN and HeLa cells. (B) K17 and P63 expression in cultures of NUC, CIN and HeLa cells. Scale bar, $100 \mu \mathrm{m}$. NUC, normal uterine cervix; CIN, cervical intraepithelial neoplasia; K, keratin.

CIN cell subcultures. Few differences in the cell growth behavior were observed in the subcultures compared with the primary cultures. The majority of CIN cells were attached to the plastic bottles within $24 \mathrm{~h}$ of being placed in the subculture. The keratinocytes in the subcultures grew faster than those in the primary cultures and reached $80 \%$ confluency in $\sim 10$ days. The CIN subcultures in each passage did not notably vary from those obtained from the primary cultures and could be passaged up to four times (Fig. 2D). During this culture period, the CIN keratinocytes maintained an unequally sized, abnormally shaped morphology, and no differentiation occurred.

Identification of high-grade CIN cells. Diffuse and strong cytoplasmic immunostaining for K14 and K19 was obtained 
in the majority of the cultured high-grade CIN and NUC epithelial cells (Fig. 3A). The results indicate that the isolated cells were cervical epithelial cells.

In addition, cytoplasmic staining for K17 and nuclear staining for P63 were observed in the majority of the cultured high-grade CIN cells and HeLa cells; however, the majority of NUC cells did not express K17 and P63 (Fig. 3B), which are two markers of cervical stem cells. The cells cultured in the present study demonstrated the specificity of high-grade CIN cells.

\section{Discussion}

To the best of our knowledge, the present study is the first to demonstrate that CIN keratinocytes may be cultured from small sections of neoplastic tissue, without a fibroblast feeder layer, and overcame the major issue that the size of neoplastic tissue is usually extremely small, which poses a challenge when attempting the isolation of an adequate number of CIN keratinocytes for culturing purposes.

Firstly, the small tissues were digested using $0.2 \%$ type I collagenase. However, unlike a previous study (17), the isolated cells in the present study were not filtered through a mesh, but seeded into 6-well flat-bottomed culture plastic plates directly, which may cause the cells that are removed during filtering to be retained, therefore resulting in an increased number of CIN keratinocytes. In addition, FBS is frequently added to the basal medium as a source of certain nutrients and growth factors (including plasma proteins, peptides, growth factors and hormones) that are essential for cell attachment and growth. However, previous studies indicate that FBS may trigger cell differentiation $(11,14-16)$. In the present study, the FBS supplemented with K-SFM was replaced with K-SFM at the first medium change, which demonstrated that the CIN keratinocytes cultured in K-SFM conditions resist terminal differentiation for at least four passages, and that fibroblast contamination was also avoided in K-SFM.

Additionally, the first medium change usually occurs on day 3. However, a previous study reported that epithelial cells exhibit a slower anchorage ability than fibroblasts, and that the reseeding of the unattached cells from the first seeded flasks into new flasks is highly advantageous (11). In the present study, the cell viability was tested prior to seeding and the viability of the unattached cells was tested on days 3,5 and 7. The results indicated that, although the viability of the unattached cells decreased to $88.00 \pm 0.50 \%$ on day 3 , the ability of cells to attach remained higher than if the unattached cells were not reseeded. The cell attachment rate increased significantly between days 3 and 5, and became relatively stable between days 5 and 7, which may be associated with the significantly decreased cell viability between days 5 and 7 . Therefore, day 5 or 6 was deduced to be the appropriate time for the first medium change for primary cultures, as the prolonged first medium change time may prevent numerous unattached CIN cells from being removed by the routine first medium change.

In the study by Bononi et al, the CIN cells were grown for a 5-week culture period and the subcultures were grown for 4 weeks (11). However, the primary culture period of high-grade CIN keratinocytes in the present study was only
12-14 days and the subcultures only required 10 days to grow. Therefore, the method used in the present study was rapid in comparison and may save time. In addition, since the medium only requires changing twice a week, the risk of exogenous microbial contamination is highly reduced using the present method.

There are few studies on the in vitro keratin expression profile of CIN and normal keratinocytes (11,12). Keratin 5 (K5), K14 and K19 are known to be associated with the basal and parabasal layers of the normal ectocervical epithelium $(11,12,22)$. However, cultured NUC keratinocytes have exhibited weak staining for K5 in a previous study (17). Therefore, only K14 and K19 were tested in the present study, and strong cytoplasmic immunostaining for K14 and K19 was obtained in the majority of high-grade CIN keratinocytes. Additionally, P63 and K17 are specific markers of cervical stem cells (22-24), and staining of the markers was detected in the majority of the cultured CIN cells. In addition, the passaged keratinocytes remained HPV-positive; therefore, the CIN keratinocytes cultured in the present study were considered to originate from cervical stem cells, and did not change from the original characteristics in the in vitro culture.

Several important limitations of the present study may be acknowledged. As few studies have focused on the in vitro keratin expression profile of CIN keratinocytes, few direct fluorescent antibodies for K17 and P63 are available. Therefore, performing flow cytometry to detect the purity of the high-grade CIN keratinocytes was not possible in the present study. Additionally, the high-grade CIN keratinocytes were only described based on cell morphology and the presence of cervical stem cell markers. Thus, additional studies are required to explore the characteristics of these keratinocytes.

In conclusion, a simple and practical method to obtain high-grade CIN keratinocytes from small neoplastic tissue fragments was set up. Compared with previous CIN culture methods, the present protocol is rapid, simple, effective and practical. In addition, the present culture method retains the HPV-CIN keratinocyte characteristics and resists cell differentiation. The cell morphology and keratin immunofluorescence staining results lead to the conclusion that the cells that were cultured in the present study were high-grade CIN keratinocytes. The present protocol is promising as cervical precancerous lesions are an important topic worldwide, and high-grade keratinocytes are crucial for the study of these lesions. The results of the present study provide an experimental basis for a variety of research purposes, particularly the additional study of the biological characteristics of high-grade CIN keratinocytes and the therapeutic methods for the treatment of precancerous lesions of the cervix.

\section{Acknowledgments}

The present study was supported by the Chinese Nature Science Foundation (grant no. 81072122).

\section{References}

1. Ferlay J, Shin HR, Bray F, Forman D, Mathers C and Parkin DM: Estimates of worldwide burden of cancer in 2008: GLOBOCAN 2008. Int J Cancer 127: 2893-2917, 2010. 
2. Stewart BW and Kleihues P (eds): Cancers of the female reproductive tract. In: World Cancer Report 2003. IARC Press, Lyon, p215, 2003.

3. Martin CM and O'Leary JJ: Histology of cervical intraepithelial neoplasia and the role of biomarkers. Best Pract Res Clin Obstet Gynaecol 25: 605-615, 2011.

4. Sellors JW and Sankaranarayanan R (eds): Colposcopy and Treatment of Cervical Intraepithelial Neoplasia: A Beginners' Manual. IARC Press, Lyon, pp13-20, 2003.

5. Wright TC Jr, Cox JT, Massad LS, Carlson J, Twiggs LB and Wilkinson EJ; American Society for Colposcopy and Cervical Pathology: 2001 consensus guidelines for the management of women with cervical intraepithelial neoplasia. Am J Obstet Gynecol 189: 295-304, 2003.

6. McCredie MRE, Sharples KJ, Paul C, Baranyai J, Medley G, Jones RW and Skegg DC: Natural history of cervical neoplasia and risk of invasive cancer in women with cervical intraepithelial neoplasia 3: A retrospective cohort study. Lancet Oncol 9: 425-434, 2008

7. Walboomers JM, Jacobs MV, Manos MM, Bosch FX, Kummer JA, Shah KV, Snijders PJ, Peto J, Meijer CJ and Muñoz N: Human papillomavirus is a necessary cause of invasive cervical cancer worldwide. J Pathol 189: 12-19, 1999.

8. Schiffman M, Castle PE, Jeronimo J, Rodriguez AC and Wacholder S: Human papillomavirus and cervical cancer. Lancet 370: 890-907, 2007.

9. Muñoz N, Castellsagué X, de González AB and Gissmann L: Chapter 1: HPV in the etiology of human cancer. Vaccine 24 (Suppl 3): S3/1-10, 2006.

10. Wimmer E, Mueller S, Tumpey TM and Taubenberger JK: Synthetic viruses: A new opportunity to understand and prevent viral disease. Nat Biotechnol 27: 1163-1172, 2009.

11. Bononi I, Bosi S, Bonaccorsi G, Marci R, Patella A, Ferretti S, Tognon M, Garutti P and Martini F: Establishment of keratinocyte colonies from small-sized cervical intraepithelial neoplasia specimens. J Cell Physiol 227: 3787-3795, 2012.

12. Stanley MA: Culture of Human Cervical Epithelial Cells. In: Culture of Epithelial Cells. Freshney RI and Freshney MG (eds). 2nd edition. Wiley-Liss, New York, NY, pp137-169, 2002.

13. Coolen NA, Verkerk M, Reijnen L, Vlig M, van den Bogaerdt AJ, Breetveld M, Gibbs S, Middelkoop E and Ulrich MMW: Culture of keratinocytes for transplantation without the need of feeder layer cells. Cell Transplant 16: 649-661, 2007.

14. Ma XL, Que YH, Kong J, Liu HQ and Zhang JS: Effect of fetal bovine serum on the proliferation and differentiation of murine corneal epithelial cells in vitro. Int J Ophthalmol 9: 817-819, 2009.
15. Lechner JF, Haugen A, McClendon IA and Shamsuddin AM: Induction of squamous differentiation of normal human bronchial epithelial cells by small amounts of serum. Differentiation 25: 229-237, 1984.

16. Bettiol E, Sartiani L, Chicha L, Krause KH, Cerbai E and Jaconi ME: Fetal bovine serum enables cardiac differentiation of human embryonic stem cells. Differentiation 75: 669-681, 2007.

17. Liu YZ, Lü XP, Pan ZX, Zhang W, Chen ZR, Wang H, Liu H and Zhang YZ: Establishment of a novel method for primary culture of normal human cervical keratinocytes. Chin Med J (Engl) 126: 3344-3347, 2013.

18. Horvat R, Herbert A, Jordan J, Bulten J and Wiener HG: Techniques and quality assurance guidelines for histopathology. In: European guidelines for quality assurance in cervical cancer screening. Arbyn M, Anttila A, Jordan J, Ronco G, Schenck U, Segnan N, Wiener HG, Herbert A, Daniel J and von Karsa L (eds). 2nd edition. IARC Press, Lyon, pp171-189, 2008.

19. Altman SA, Randers L and Rao G: Comparison of Trypan Blue dye exclusion and fluorometric assays for mammalian cell viability determinations. Biotechnol Prog 9: 671-674, 1993.

20. Gravitt PE, Peyton CL, Alessi TQ, Wheeler CM, Coutlée F, Hildesheim A, Schiffman MH, Scott DR and Apple RJ: Improved amplification of genital human papillomaviruses. J Clin Microbiol 38: 357-361, 2000.

21. Liu X, Zhang S, Ruan Q, Ji Y, Ma L and Zhang Y: Prevalence and type distribution of human papillomavirus in women with cervical lesions in Liaoning Province, China. Int J Gynecol Cancer 20: 147-153, 2010.

22. Quade BJ, Yang A, Wang Y, Sun D, Park J, Sheets EE, Cviko A, Federschneider JM, Peters R, McKeon FD and Crum CP: Expression of the p53 homologue p63 in early cervical neoplasia. Gynecol Oncol 80: 24-29, 2001.

23. Martens JE, Arends J, Van der Linden PJ, De Boer BA and Helmerhorst TJ: Cytokeratin 17 and p63 are markers of the HPV target cell, the cervical stem cell. Anticancer Res 24 (2B): 771-775, 2004

24. Ikeda K, Tate G, Suzuki T and Mitsuya T: Coordinate expression of cytokeratin 8 and cytokeratin 17 immunohistochemical staining in cervical intraepithelial neoplasia and cervical squamous cell carcinoma: An immunohistochemical analysis and review of the literature. Gynecol Oncol 108: 598-602, 2008. 